\title{
Adaptive-Beamforming-Based Multiple Targets Signal Separation
}

\author{
Zhe Wei, Jianguo Huang, Yuejiao Hui \\ College of Marine Engineering \\ Northwestern Polytechnical University \\ Xi'an, Shaanxi, China, 710072
}

\begin{abstract}
In practical array signal processing systems, how to separate the required target signal from the received mixed signals effectively is an important research work. Firstly, for the conventional diagonal loading approaches, it is difficult to choose the DL level reliably. To solve this problem, by using the shrinkage method we present a General-Linear-CombinationBased robust adaptive beamformer (GLC) in this paper. Secondly, different from the traditional time-domain and frequency-domain separation methods, we innovatively apply the adaptive beamforming technique into the research of multiple targets signal separation in this paper, and give the specifications quantitatively to measure the separation effects: spatial separation angle and similarity coefficient. Simulation results show that compared with traditional methods, the GLC-based method has smaller spatial separation angle and greater similarity coefficient, which could separate the required target signal more accurately.
\end{abstract}

Keywords- signal separation; adaptive beamforming; shrinkage method; diagonal loading

\section{INTRODUCTION}

Beamforming is one of the most important branches in modern array signal processing. It has been used in so many areas as radar, sonar, astronomy, seismology, medical imaging and wireless communications [1]. Beamforming technology uses the spatial information of the signals received by the sensor array to extract the desired signal. It can be divided into conventional beamforming $(\mathrm{CBF})$ and adaptive beamforming (ABF) [2]. However, in practice there may be multiple targets appeared within the beam searching plane or the targets may cast interferences to countermeasure. At this point the received signal is the mixture of multiple target signals, and this requires the receiving array to accurately separate the multiple signals, and then make feature extraction of these targets respectively for the purpose of recognition and anti-jamming. Currently there have been a lot of time-domain and frequency-domain signal processing methods [3] applied to multiple targets signal separation. But when the multiple target signals are at the same frequency range or the targets cast interference to countermeasure, these methods can not accurately separate the desired target signal [4]. While the adaptive beamforming technique could effectively enhance or inhibit the signals from different spatial directions, thus well separate and extract the desired target signal.

However, in practice affected by limited snapshots and observation direction errors, the covariance matrix could not be estimated accurately and there would also be some errors in the array steering vector, which caused the performance of the traditional Standard Capon Beamformer (SCB) [5] degrade significantly [6]. In recent years, Jian $\mathrm{Li}$ et al proposed the Robust Capon beamforming algorithm (RCB) [7] based on the steering vector uncertainty. And on this basis, by imposing constraint on the module of the steering vector, they proposed the Doubly Constrained Robust Capon Beamforming algorithm (DCRCB) [8].

In fact, the algorithms mentioned above are all based on the user setting parameters, whereas the General-LinearCombination-Based [9] robust adaptive beamformer (GLC) focused in this paper could completely automatically compute the DL level without specifying any user parameters, which is a good solution to the problem aforementioned that it is difficult to choose the DL level reliably. In addition, in this paper we innovatively apply the adaptive beamforming technique into multiple targets signal separation, and give the specifications to measure the separation effects. By comparison of the application of CBF, SCB and GLC beamformers in the signal separation, it is obvious to see the performance advantage of the GLC method. Theoretical analysis and computer simulations verify the correctness and effectiveness of this method.

\section{STANDARD CAPON BEAMFORMER}

Consider a Uniform Linear Array (ULA) comprising $\mathrm{N}$ sensors and let $\mathbf{R}$ denote the theoretical covariance matrix of the array output vector. We assume that $\mathbf{R}>0$ (positive definite) has the following form:

$$
\mathbf{R}=E\left[\mathbf{X}(t) \mathbf{X}^{\mathrm{H}}(t)\right]=\mathbf{A} \mathbf{R}_{\mathrm{S}} \mathbf{A}^{\mathrm{H}}+\sigma_{n}^{2} \mathbf{I}
$$

where $\mathbf{A}=\left[\mathbf{a}\left(\theta_{0}\right), \mathbf{a}\left(\theta_{1}\right), \ldots, \mathbf{a}\left(\theta_{\mathrm{P}}\right)\right], \mathbf{a}\left(\theta_{0}\right)$ and $\mathbf{a}\left(\theta_{i}\right), \quad(i=1,2, \ldots, \mathbf{P})$ are the steering vectors of the target signal and $\mathrm{P}$ interferences respectively. $\sigma_{n}^{2}$ denotes the noise power, $\mathbf{R}_{\mathrm{S}}$ is the autocorrelation matrix of the signal complex envelope.

But in practice the accurate covariance $\mathbf{R}$ is unavailable, so the sample covariance $\hat{\mathbf{R}}$ is always used to replace $\mathbf{R}$

$$
\hat{\mathbf{R}}=\frac{1}{\mathrm{~K}} \sum_{i=1}^{\mathrm{K}} \mathbf{X}(i) \mathbf{X}^{\mathrm{H}}(i)
$$

where, $\mathrm{K}$ denotes the snapshot number and $\mathbf{X}(i)$ denotes the $i$ th snapshot.

The SCB beamformer determines the array weight vector by minimizing the array output power subject to the constraint that the signal is passed undistorted. The mathematical expression is as follows:

This work was supported in part by the National Science Foundation of China under Grant No. 60972152, and by the National Laboratory Foundation of China under Grant No. 9140C230309110C23. 
The solution to (3) is

$$
\begin{aligned}
& \min _{\mathbf{w}} \mathbf{w}^{*} \mathbf{R w} \\
& \text { s.t. } \quad \mathbf{w}^{*} \mathbf{a}_{0}=1
\end{aligned}
$$

$$
\mathrm{w}_{\mathrm{SCB}}=\frac{\mathbf{R}^{-1} \mathbf{a}_{0}}{\mathbf{a}_{0}^{*} \mathbf{R}^{-1} \mathbf{a}_{0}} .
$$

When the snapshots are limited and the covariance cannot be estimated accurately, even without the array steering vector error, the performance of the SCB beamformer will degrade significantly.

\section{GENERALIZED-LINEAR-COMBINATION-BASED ROBUST CAPON BEAMFORMER}

\section{A. Generalized-Linear-Combination-Based Covariance Matrix Estimation}

The sample covariance matrix $\hat{\mathbf{R}}$ is not a good estimate for the theoretical covariance matrix $\mathbf{R}$ when the snapshots are fewer (relative to the array number). To alleviate this problem, by using the shrinkage method [10], we present a GLC-based covariance estimation. We consider a combination of the sample covariance matrix $\hat{\mathbf{R}}$ and identity matrix $\mathbf{I}$ by the GLC form to obtain a more accurate estimate of $\mathbf{R}$ :

$$
\widetilde{\mathbf{R}}=\alpha \mathbf{I}+\beta \hat{\mathbf{R}}
$$

where, $\widetilde{\mathbf{R}}$ satisfies $\widetilde{\mathbf{R}} \geq 0$ (positive semidefinite), which is a more accurate estimate of $\mathbf{R} . \alpha$ and $\beta$ are the shrinkage parameters, which are chosen by minimizing the MSE of $\widetilde{\mathbf{R}}$, as stated in $[10]$ that $\operatorname{MSE}(\widetilde{\mathbf{R}})=E\left\{\mid \widetilde{\mathbf{R}}-\mathbf{R} \|^{2}\right\}$. We restrict $\alpha \geq 0, \beta \geq 0$ to guarantee that $\widetilde{\mathbf{R}} \geq 0$.

Next we consider the GLC-based MSE minimization problem and calculate the shrinkage parameters.

$$
\begin{aligned}
& \operatorname{MSE}(\widetilde{\mathbf{R}})=E\left\{\|\alpha \mathbf{I}-(1-\beta) \mathbf{R}+\beta(\hat{\mathbf{R}}-\mathbf{R})\|^{2}\right\} \\
& =\|\alpha \mathbf{I}-(1-\beta) \mathbf{R}\|^{2}+\beta^{2} E\left\{\|(\hat{\mathbf{R}}-\mathbf{R})\|^{2}\right\} \\
& =\alpha^{2} N-2 \alpha(1-\beta) \operatorname{tr}(\mathbf{R})+(1-\beta)^{2}\|\mathbf{R}\|^{2} \\
& +\beta^{2} E\left\{\|(\hat{\mathbf{R}}-\mathbf{R})\|^{2}\right\}
\end{aligned}
$$

Therefore, the optimal values of $\alpha$ and $\beta$ (unconstrained) are

$$
\begin{aligned}
& \beta_{0}=\frac{\gamma}{\rho+\gamma} \\
& \alpha_{0}=v\left(1-\beta_{0}\right)=v \frac{\rho}{\gamma+\rho}
\end{aligned}
$$

where $\rho \stackrel{\text { def }}{=} E\left\{\|(\hat{\mathbf{R}}-\mathbf{R})\|^{2}\right\}, \quad v=\operatorname{tr}(\mathbf{R}) / N, \quad \gamma=\|\boldsymbol{I}-\mathbf{R}\|^{2}$. We notice that $\beta_{0} \in[0,1], \quad \alpha_{0} \geq 0$, and that $\alpha_{0}$ and $\beta_{0}$ are all depended on the unknown covariance $\mathbf{R}$. In order to estimate $\alpha_{0}$ and $\beta_{0}$ by the given data, we need to estimate the value of $\rho$ at first, the specific value of $\rho$ can be calculated by the following equation [11]:

$$
\hat{\rho}=\frac{1}{\mathrm{~K}^{2}} \sum_{i=1}^{\mathrm{K}}\|\mathbf{X}(i)\|^{4}-\frac{1}{\mathrm{~K}}\|\hat{\mathbf{R}}\|^{2}
$$

In addition we have

$$
\gamma+\rho=\|v \mathbf{I}-\mathbf{R}\|^{2}+E\left\{\|\hat{\mathbf{R}}-\mathbf{R}\|^{2}\right\}=E\left\{\|\hat{\mathbf{R}}-v \mathbf{I}\|^{2}\right\}
$$

an estimate of which is given by $\|\hat{\mathbf{R}}-\hat{\mathbf{V}}\|^{2}$, where $\hat{v}$ can be acquired by replacing $\mathbf{R}$ by its unbiased estimate $\hat{\mathbf{R}}$. Then we could get the estimate of $\alpha_{0}$ and $\beta_{0}$.

$$
\begin{aligned}
& \hat{\alpha}_{0}=\hat{v} \frac{\hat{\rho}}{\|\hat{\mathbf{R}}-\hat{v} \mathbf{I}\|^{2}} \\
& \hat{\beta}_{0}=1-\frac{\hat{\rho}}{\|\hat{\mathbf{R}}-\hat{\mathbf{I}}\|^{2}}
\end{aligned}
$$

The estimate of $\beta_{0}$ in (12) may be negative. To guarantee the estimate of $\beta_{0}$ nonnegative, as stated in [10], we could use the following estimate of $\alpha_{0}$ :

$$
\hat{\alpha}_{0}=\min \left[\hat{v} \frac{\hat{\rho}}{\|\hat{\mathbf{R}}-\hat{\boldsymbol{V}}\|^{2}}, \hat{v}\right]
$$

the corresponding estimate of $\beta_{0}$ is:

$$
\hat{\beta}_{0}=1-\frac{\hat{\alpha}_{0}}{\hat{v}} \text {. }
$$

It can be seen that (13) and (14) indirectly guarantee that $\hat{\alpha}_{0} \geq 0, \hat{\beta}_{0} \geq 0$.

\section{B. Generalized-Linear-Combination-Based Robust Capon Beamformer}

From the previous discussion, we have obtained a more accurate estimate $\widetilde{\mathbf{R}}$ for the covariance matrix $\mathbf{R}$. Actually it is clear that $\widetilde{\mathbf{R}}$ has the diagonal loading form. The specific expression of $\widetilde{\mathbf{R}}$ is as follows:

$$
\widetilde{\mathbf{R}}=\hat{\alpha}_{0} \mathbf{I}+\hat{\beta}_{0} \hat{\mathbf{R}}
$$

where $\hat{\alpha}_{0}$ and $\hat{\beta}_{0}$ can be obtained from (13) and (14) respectively. Using $\widetilde{\mathbf{R}}$ in place of the sample covariance $\hat{\mathbf{R}}$ in the SCB formulation yields the weight vector of the GLCbased robust adaptive beamformer:

$$
\mathbf{w}_{\mathrm{GLC}}=\frac{\widetilde{\mathbf{R}}^{-1} \mathbf{a}}{\mathbf{a}^{\mathrm{H}} \widetilde{\mathbf{R}}^{-1} \mathbf{a}} .
$$

Rewriting (16) as follows (assuming that $\hat{\beta}_{0} \neq 0$ ):

$$
\widetilde{\mathbf{w}}_{\mathrm{GLC}}=\frac{\left[\frac{\hat{\alpha}_{0}}{\hat{\beta}_{0}} \mathbf{I}+\hat{\mathbf{R}}\right]^{-1} \mathbf{a}}{\mathbf{a}^{\mathrm{H}}\left[\frac{\hat{\alpha}_{0}}{\hat{\beta}_{0}} \mathbf{I}+\hat{\mathbf{R}}\right]^{-1} \mathbf{a}}
$$

The signal power estimate is:

$$
\hat{\sigma}_{0}^{2}=\mathbf{w}_{\mathrm{GLC}}^{\mathrm{H}} \widetilde{\mathbf{R}}_{\mathrm{GLC}} \mathbf{w}_{\mathrm{GLC}}=\frac{1}{\mathbf{a}^{*} \widetilde{\mathbf{R}}_{\mathrm{GLC}} \mathbf{a}}
$$

We observe that the GLC-based robust adaptive beamformer is actually a diagonal loading approach, but compared to various diagonal loading approaches proposed 
before, its biggest advantage is that the DL level can be easily and automatically computed from the observed data snapshots $\{\mathbf{X}(i)\}_{i=1}^{\mathrm{K}}$, not like other approaches which require the users to set the corresponding parameters. It makes the calculation of the DL level in practice much easier, improves the efficiency and ensures the stability of the approach.

\section{Principle of Multiple Targets Signal SeParation}

Assuming the target signal that the receiving array received is the mixture of D target signals $s_{1}, s_{2}, \ldots, s_{\mathrm{D}}$. The method of multiple targets signal spatial separation is that: Firstly, make DOA estimation of the D target signals. Then make each DOA as the expected direction and do the beamforming. Finally, take turns to pass the mixed target signals through the D different array beams, then we get the estimates of D target signals $\hat{S}_{1}, \hat{S}_{2}, \ldots, \hat{S}_{\mathrm{D}}$. The specific block diagram is shown in Fig. 1.

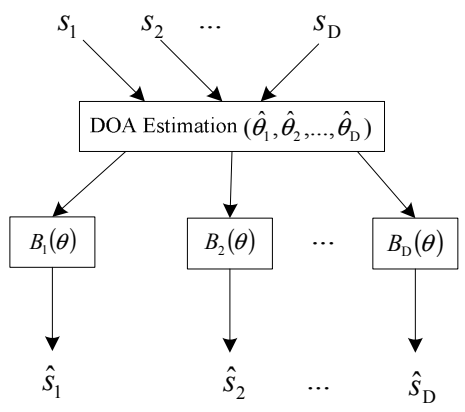

Figure 1. Schematic diagram of multiple targets signal separation

To quantitatively analyze the effects of multiple targets signal separation, we present the following specifications:

1. Spatial Separation Angel: the minimum target angel interval that can be separated.

2 Similarity Coefficient: the correlation coefficient between the separated and the original target signals, the computing formula is as follows:

$$
\gamma_{x_{i} y_{i}}=\frac{\operatorname{cov}\left(x_{i}, y_{i}\right)}{\sqrt{\mathrm{D} x_{i} \mathrm{D} y_{i}}}
$$

where $\operatorname{cov}\left(x_{i}, y_{i}\right)$ is the cross-correlation function between the original target signal $x_{i}$ and the separated target signal $y_{i}$, $\mathrm{D} x_{i}$ and $\mathrm{D} y_{i}$ denote the variance of $x_{i}$ and $y_{i}$ respectively. When the separated signal is identical with the original signal, the similarity coefficient is 1 . The smaller difference between the separated and the original target signal, the larger the similarity coefficient is, and the better separation effect it will be.

\section{Simulations And Performance ANALysis}

We present below several numerical examples that compare the performance of the $\mathrm{CBF}, \mathrm{SCB}$ and GLC beamformers applied in multiple targets signal separation to verify the superiority of the GLC-based approach. Consider a ULA composed of $\mathrm{M}=10$ ideal array elements, the array element spacing is half wavelength. The DOA of target signal 1 is 0 degree, and the signal power is $0 \mathrm{~dB}$. Target signal 2 is random noise with the DOA 20 degree and signal power $20 \mathrm{~dB}$. Background noise is the Gaussian white noise with mean 0 and variance 1 . Target signal 1, target signal 2 and the background noise are mutually independent. The sampling frequency is 10 $\mathrm{Hz}$ and the snapshots are 200.

Simulation 1 Comparison of the CBF, SCB and GLC beamformers separated waveforms

Assuming there is no array steering vector error. Fig. 2 shows the contrast of the different beampattens of the three beamformers. From Fig. 2, we observe that in the direction of strong interference target signal 2 the SCB and GLC beamformers could form deep nulls, and thus effectively suppress the interference. Meanwhile, compared with SCB GLC has better performance that it has lower sidelobes and produces deeper nulls $(-50 \mathrm{~dB})$ in the interference direction, so it is able to suppress the strong interference better and improve the output SINR.

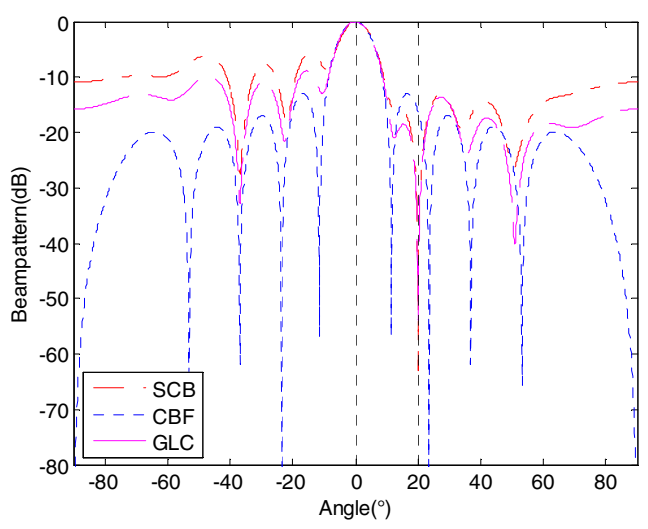

Figure 2. Comparison of $\mathrm{CBF}, \mathrm{SCB}$ and GLC beampatterns

The comparison of target 1 separated and original signal waveforms is given in Fig. 3. It can be seen that SCB and GLC could both well separate the desired target signal. After calculation we obtain the similarity coefficients between the separated and the original signals by using CBF, SCB and GLC respectively. The similarity coefficients are $0.5252,0.9295$ and 0.9530 . Obviously GLC has better performance.
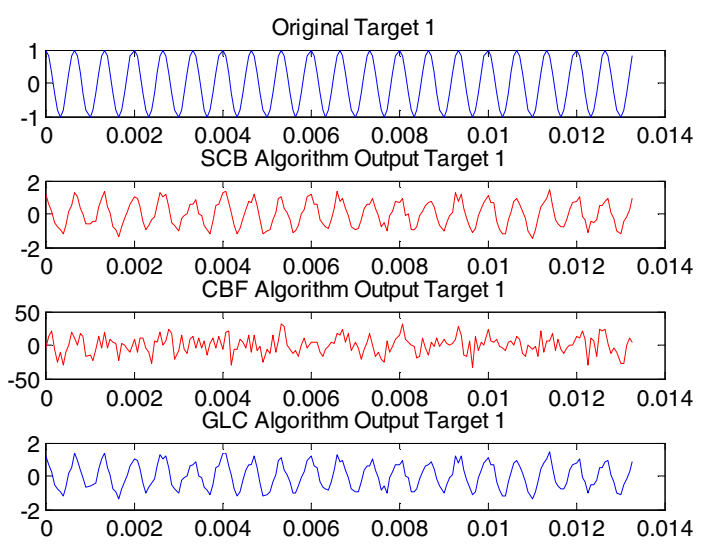

Figure 3. Comparison of target 1 separated and original signal waveforms 
Simulation 2 Influence of target angle interval on the similarity coefficient of target 1

Fig. 4 shows the curve of the similarity coefficient of the separated and the original signals versus different target angle intervals of target 1 by using the three different beamformers. From the figure we can observe that no matter what the target angle intervals are, the separation performance of $\mathrm{CBF}$ is relatively poor. However, for SCB and GLC with the increase of the target angle interval, the similarity coefficients gradually approximate to 1 and the separation effects attain to the best. When the target angle interval is greater than 2.5 degree, the similarity coefficients of the separated and the original signals of SCB and GLC have reached above 0.7, which have good separation effects. When the target angle interval is greater than 6 degree, the similarity coefficients reach above 0.9 , which means that the desired target signal 1 can be effectively separated. Compared with SCB, GLC has better separation effect.

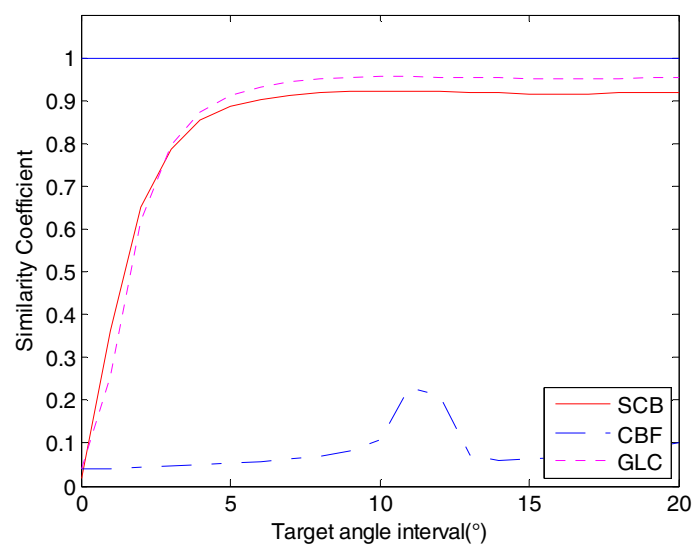

Figure 4. Curve of target 1 similarity coefficient versus target angle interval

Simulation 3 Influence of SNR on the similarity coefficient of target 1 with given target angle interval

In the same simulation conditions, the input SNR varies from $-20 \mathrm{~dB}$ to $20 \mathrm{~dB}$. Fig. 5 shows the curve of the similarity coefficient between the separated and the original signals of target 1versus SNR. It can be seen from the figure that with the SNR increasing, the similarity coefficients of the separated and the original signals of target 1 gradually increase. When SNR reaches above $-2 \mathrm{~dB}$ the similarity coefficients reach above 0.9 , which means that the desired target signal 1 can be effectively separated. The GLC method has obvious advantage.

\section{CONCLUSIONS}

We have considered a GLC-based beamformer in this paper, through improving the conventional sample covariance a more accurate estimate has been obtained. Compared with other traditional methods the new method could more effectively suppress the interference, improve the output SINR, especially in the situation of fewer snapshots the advantages are more obvious. The most important thing is that this method could completely automatically compute the DL level without specifying any user parameters. In addition, in this paper we have focused on the principle and method of spatial multiple targets signal separation, proposed the corresponding specifications, and applied the adaptive beamforming techniques into it. Numerical examples have shown that compared with other methods the new method has smaller spatial separation angle and greater similarity coefficient under the same conditions, so the desired signal can be more accurately separated from the mixed signals

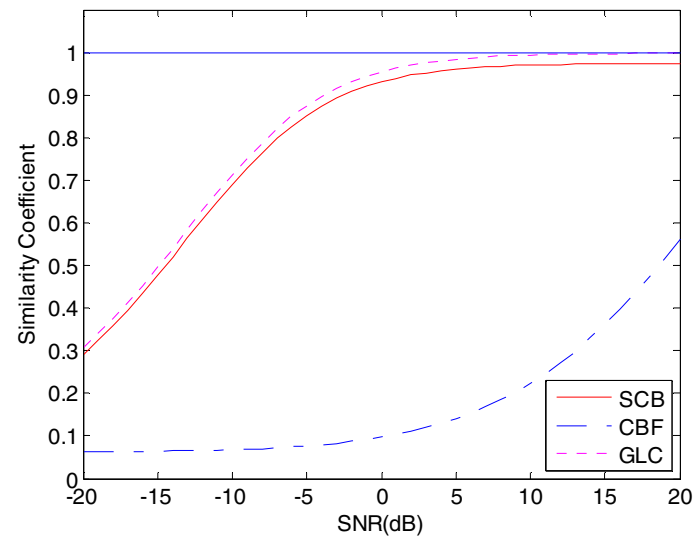

Figure 5. Curve of target 1 similarity coefficient versus SNR

\section{ACKNOWLEDGMENT}

The author would like to thank Huawei Yang for help in the research, Hai Huang and Huihui Wang in English wring.

\section{REFERENCES}

[1] H. L. Van Trees, Detection, Estimation, and Modulation Theory, Part IV, Optimum Array Processing. New York: Wiley, 2002.

[2] Jinkuan Wang, and Xin Song, Robust Adaptive Array Signal Processing, Beijing: Publishing House of Electronics Industry, 2009

[3] A. V. Oppenheim, R. W. Schafer, and J. R. Buck, Discrete-Time Signal Processing (2nd Edition). NJ: Prentice-Hall, 1999.

[4] Huawei Yang, Study on Underwater Adaptive Beamforming and Signal Separation Technique, Xi'an: Northwestern Plolytechnical University, 2010.

[5] J. Capon, "High resolution frequency-wave number spectrum analysis," Proceedings of IEEE, vol. 57, pp. 1408-1418, Aug. 1969.

[6] Congfeng Liu, Xiaojun Liu, and Guisheng Liao, "Review for Robust Adaptive Beamforming Algorithms," Journal of CAEIT, vol. 4, no. 6, pp: 560-565, Dec. 2009.

[7] Jian Li, P. Stoica, and Zhisong Wang, "On robust Capon beamforming and diagonal loading," IEEE Transactions on Signal Processing, vol. 51, no. 7, pp. 1702-1715, July 2003.

[8] Jian Li, P. Stoica, and Zhisong Wang, "Doubly Constrained Robust Capon Beamformer," IEEE Transactions on Signal Processing, vol. 52, no. 9, pp. 2407-2423, July 2004.

[9] Lin Du, Jian Li, and P. Stoica, "Fully Automatic Computation of Diagonal Loading Levels for Robust Adaptive Beamforming," IEEE Transactions on Aerospace and Electronic System, vol. 46, no. 1, pp. 449-458, Jan. 2010.

[10] O. Ledoit, and M. Wolf, "A well-conditioned estimator for largedimensional covariance matrices," Journal of Multivariate Analysis, vol. 88, pp: 365-411 2004.

[11] P. Stoica, Jian Li,, Xumin Zhu, and J. R. Guerci, "On using a priori knowledge in space-time adaptive processing," IEEE Transactions on Signal Processing, vol. 56, no. 6, pp. 2598-2602, June 2008. 